\title{
Population based epidemiology and prognosis of mesothelioma in Leeds, UK
}

\author{
A Chapman, S Mulrennan, B Ladd, M F Muers
}

Leeds Teaching Hospitals NHS Trust, Leeds, UK

Correspondence to: Dr A L Chapman, The Friarage Hospital Northallerton,

Northallerton, North Yorkshire DL6 1JG, UK; alexander chapman@stees.nhs.uk

Received 20 March 2007 Accepted 12 December 2007

Published Online First

17 January 2008

\section{ABSTRACT}

Introduction: Malignant mesothelioma is a fatal neoplasm, which is rapidly increasing in incidence throughout Western Europe. To date there have been no studies reporting on the natural history and interventional practices on a comprehensive unselected population, as opposed to reports from referral institutions or compensation claimants. We present a population based study capturing data on all patients with mesothelioma presenting within a defined geographical area over a 4 year period in the UK.

Method: Data of all cases occurring in Leeds with a population of 750000 were collected retrospectively from 2002 to 2003 and prospectively from 2004 to 2005 . All patients' hospital records and the Trust histology database were reviewed, as well as coroner's reports on all patients with a post mortem diagnosis of mesothelioma.

Results: Over the 4 year study period, there were a total of 146 cases in Leeds; $77 \%$ were male. Median age was 74 years (range 36-93). Median survival from diagnosis was 8.9 months. $92 \%$ and $8 \%$ had histological or cytological confirmation, respectively. $85 \%$ had documented evidence of definite or probable exposure to asbestos. 110/146 (75\%) had symptomatic pleural effusions at presentation. Twice the number of patients (42 vs 17) were managed with surgical rather than bedside pleurodesis and these had a lower recurrence rate ( $14 \%$ vs $47 \% ; p=0.02) .122$ patients had video assisted thoracoscopic surgery/cutting CT biopsies or chest drains. 73/122 (60\%) had prophylactic radiotherapy to these sites. There were seven cases $(5 \%)$ of tract invasion by tumour and six of these had received prophylactic radiotherapy. Median time to seeding was 174 days. 92/146 (63\%) had a performance status of 2 or better at diagnosis but only 54/146 were considered fit for chemotherapy. Of these, 28 (52\%) declined chemotherapy; the overall uptake of chemotherapy or entry into a trial was $18 \%$. No patient had radical surgery.

Conclusion: This comprehensive population based audit has shown that the median age at presentation of malignant mesothelioma is increasing and baseline performance status and survival is worse than in selected series. $37 \%$ of patients were considered suitable for palliative chemotherapy but less than $20 \%$ accepted this offer. Thorascopic pleurodesis appears to be associated with fewer recurrences. The role of prophylactic radiotherapy to chest drain and biopsy sites needs reappraisal.

Malignant mesothelioma is a fatal neoplasm with very few patients surviving more than 2 years from onset. ${ }^{1}$ Mortality in the UK is projected to peak at 2450 deaths per year by 2015. ${ }^{2}$ Previous studies from the UK have reported a median survival of 14 months $^{3}$ and median age at diagnosis of
64 years. ${ }^{4}$ There are no data in the literature on the overall natural history and management within a complete and unselected population from a defined geographical area.

Recent studies show that chemotherapy for malignant mesothelioma may be starting to have a clinical impact. ${ }^{5}$ A phase III study of cisplatin with pemetrexed demonstrated improved survival and response rates compared with those receiving single agent cisplatin alone. ${ }^{6}$ However, survival benefit was modest, and in their recent systematic review, Ellis et al acknowledged that advantages in survival benefit, improved symptom control and quality of life with chemotherapy over and above active supportive care (active symptom control) have yet to be established.' MSO1, a British Thoracic Society (BTS)/Medical Research Council (MRC) sponsored trial comparing two chemotherapy regimens with active symptom control, is complete and publication of their results is imminent. Current BTS recommendations state that entry into chemotherapy trials for mesothelioma should be encouraged. ${ }^{8}$

We present a population based audit capturing very nearly $100 \%$ of patients with mesothelioma presenting in a defined geographical area over a 4 year period. The audit recorded: age and performance status at presentation, survival from diagnosis, management of pleural effusion and uptake into chemotherapy trials.

\section{METHODS}

The study included all new cases of malignant mesothelioma within the boundaries of the five Leeds primary care trusts between 1 January 2002 and 31 December 2005. This well defined geographical area encompasses the 750000 population of Leeds, a conurbation in West Yorkshire, UK. All patients included in the audit were seen at the Leeds Teaching Hospitals NHS Trust as part of their diagnostic and/or management process

We retrospectively analysed all cases from 2002 to 2003 , and all cases from 2004 to 2005 were studied prospectively. Cases were identified by diagnosis from the Leeds Cancer Centre database for the retrospective portion of the audit, and from 2004 onwards were identified prospectively at regional multidisciplinary team (MDT) meetings. The audit population was then validated using the Cancer Centre database and MDT records, as well as the Trust histology database and regional coroner's records. The audit population was also compared with data held by the Northern and Yorkshire Cancer Registry and Information Service (NYCRIS) (table 1). 
Table 1 Incidence per year compared with NYCRIS mesothelioma registrations

\begin{tabular}{llll}
\hline Year & $\begin{array}{l}\text { Audit } \\
\text { population }\end{array}$ & $\begin{array}{l}\text { Incidence } \\
\text { per } \mathbf{1 0 0} \mathbf{0 0 0}\end{array}$ & NYCRIS registrations \\
\hline 2002 & 33 & 4.4 & 32 \\
2003 & 40 & 5.3 & 41 \\
2004 & 41 & 5.5 & 34 \\
2005 & 32 & 4.3 & Data not yet available \\
\hline
\end{tabular}

NYCRIS, Northern and Yorkshire Cancer Registry and Information Service.

All patients included in the audit sample were discussed by the Leeds Cancer Centre MDT and were accepted as having a diagnosis of malignant mesothelioma. In nearly all cases the diagnosis of malignant mesothelioma was made following clinical and radiological review with contrast thoracic CT and supported by thoracoscopic appearances together with histological specimens of biopsy material and immunocytochemistry of cytological specimens when present. ${ }^{9-11}$

Identified patients' records were reviewed for the audit by physicians from the Department of Respiratory Medicine, Leeds Teaching Hospitals NHS Trust. The following data were recorded from the case notes.

- World Health Organisation/Eastern Cooperative Oncology Group (WHO/ECOG) performance status at presentation. Where not formally documented, the performance status was ascertained from the other clinical details recorded.

- History of asbestos exposure, classified as definite, probable or uncertain. Probable exposure was used to describe patients in high risk occupations who did not report definite exposure.

- Incidence of symptomatic pleural effusions at presentation, and any complications of management.

- Prophylactic radiotherapy and incidence of tract invasion by tumour.

- Recruitment into chemotherapy trials.

- Survival from date of diagnosis, for this audit defined as the date of the histological/cytological sample. Patients diagnosed after death were excluded from the survival analysis.

\section{Statistical analysis}

All tests were performed using SPSS V.11.0 (SPSS Inc, Chicago, Illinois, USA). Results are presented as absolute frequencies. Survival curves were calculated using the Kaplan-Meier method and compared using the log rank test. Using this method, patients who are alive at the time of analysis are censored, meaning their survival to the date of analysis is represented in the survival curve, but their presence does not affect the cumulative survival ratio. The $\chi^{2}$ and Fisher's exact tests were used to compare categorical data. Significance was assumed at the standard $5 \%$ level.

\section{RESULTS}

\section{Population demographics}

The total number of confirmed cases in this series over a 4 year study period was 146 for a population of 750000 . The calculated incidence was 4.9 per 100000 population per year. A further four cases had a diagnosis based on clinical evidence and CT appearances; two are still alive and two have died. These patients were excluded from our population as they had no histological/cytological confirmation of mesothelioma and the diagnosis was therefore uncertain. There was no obvious increase or decrease in incidence over the study period (table 1). Median age at the time of diagnosis was 74 years (range 36-93).

\section{Diagnosis}

The diagnosis of malignant pleural mesothelioma was confirmed with a histological specimen in $91.8 \%(134 / 146)$ of the sample before or after death. Cytological evidence supported the clinical diagnosis in $8.2 \%$.

In our series, subtypes of mesothelioma were recorded in 36\% of the sample. There were 34 epithelioid, 10 sarcomatoid (of which five were desmoplastic) and nine biphasic cases identified.

\section{WHO/ECOG performance status at diagnosis}

Performance status at diagnosis was as follows: 0 (11\%), 1 (32.9\%), 2 (19.1\%), 3 (25.3\%) and 4 (7.6\%). Performance status was not recorded in six (4.1\%) patients. Mean performance status at presentation was 1.8. Patients with a performance status worse than 2 were not eligible to receive chemotherapy.

\section{Asbestos exposure}

Asbestos histories were recorded in 86\% (126/146) of cases overall. Definite asbestos exposure was reported in 57\% (72/ 126), $28 \%(35 / 126)$ reported probable exposure and 15\% (19/ 126) could not recall any previous exposure.

\section{Survival}

Survival was measured from the date of diagnosis, which for this audit was defined as the date of the histological/cytological sample. Three of 146 patients were diagnosed after death and were excluded from the survival analysis. Median survival from the time of the clinical diagnosis was 267 days ( $95 \%$ confidence interval (CI) 178 to 356 ), or approximately 8.9 months (fig 1). Median survival for the histological subtypes were 325 days ( $95 \%$ CI 240 to $410, \mathrm{n}=34$ ) for epithelioid, 175 days ( $95 \%$ CI 0 to $445, \mathrm{n}=10$ ) for sarcomatoid and 161 days ( $95 \%$ CI 93 to 229 , $\mathrm{n}=9$ ) for biphasic. Median survival for the remaining 97 cases without an identified histological subtype was 211 days (95\% CI 119 to 302$)$.

\section{Management of pleural effusion}

In our series, 110/146 (75\%) patients had symptomatic pleural effusion at presentation. This is similar to the $78 \%$ of patients with pleural effusions identified at presentation by Clayson in the north of England in her recent community based study. ${ }^{12}$ Ninety-nine of $110(90 \%)$ of those patients with a symptomatic pleural effusion received active treatment: $42 \%$ (42/99) received surgical pleurodesis; $17 \%$ (17/99) pleurodesis via a chest drain at the bedside with talc slurry (9/17), bleomycin (6/17) or tetracycline (2/17) as the pleurodesing agent; $26 \%(26 / 99)$ had a drain only; $13 \%$ had long term indwelling catheters. ${ }^{13}$ Not all patients received drainage or pleurodesis. Cited reasons included problems with blocked and dislodged drains, patient refusal or futility in a dying patient. Ten per cent (11/110) of those with symptomatic pleural effusion received no intervention or simple aspiration only.

Of the 99 patients who received active management of their pleural fluid, the total recurrence rate (as defined by an increase in breathlessness with radiological evidence of fluid re-accumulation) was $23 \%$. Patients with a surgical rather than bedside pleurodesis had a lower recurrence rate ( $14 \%$ vs $47 \%$; $p=0.02$, Fisher's exact test). Recurrence after bedside pleurodesis was 


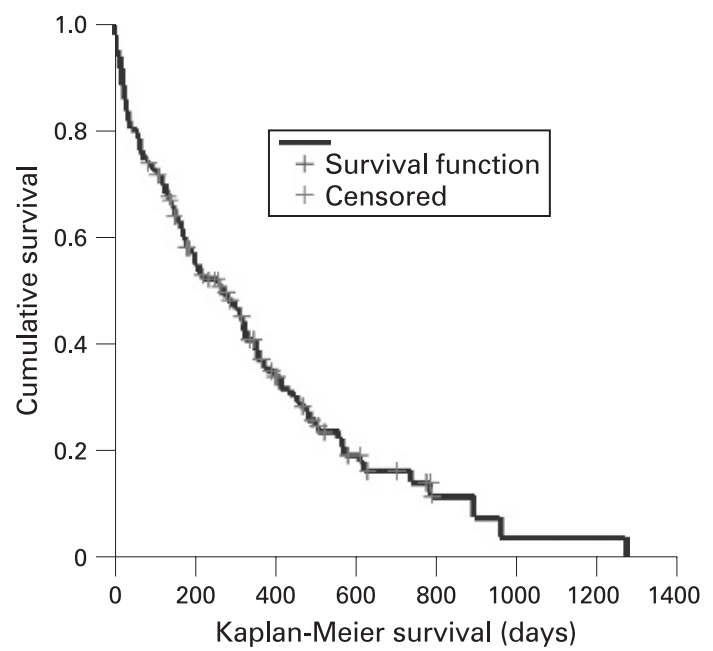

Figure 1 Survival function.

$8 / 17(47 \%)$ and after chest drain only $9 / 26(35 \%)$, and this was not statistically significant.

Prophylactic radiotherapy to prevent tract invasion with tumour A total of 122 patients had video assisted thoracoscopic surgery (VATS), cutting CT biopsy or chest drain. Overall, 60\% (73/ 122) of these patients had prophylactic radiotherapy to their intervention sites. Patients referred for prophylactic radiotherapy received three separate daily fractions of $7 \mathrm{~Gy}$. There were seven cases $(5 \%)$ overall of tract invasion by tumour and $6 / 7$ of these cases had received prophylactic radiotherapy, three of those within 6 weeks of the procedure. The median waiting time from intervention to radiotherapy was 52 days. Thus in the patients who received no radiotherapy, the incidence of metastasis was $2 \%(1 / 49)$ compared with $8 \%(6 / 73)$ in those who had ( $p=0.243$, Fisher's exact test). The median time from intervention to appearance of a metastasis at the tract site was 174 days (range 76-360).

\section{Chemotherapy}

The majority of patients who received chemotherapy were entered into the MSO1, the BTS/MRC randomised control trial comparing mitomycin, vinblastine and cisplatin together or vinorelbine alone with active symptom control (ASC) $(n=13$, three of these randomised to ASC). Earlier during the study period, seven patients were recruited into a commercially sponsored study comparing pemetrexed vs pemetrexed/cisplatin vs pemetrexed/carboplatin.

Ninety-two of 146 (63\%) patients had a performance status at presentation of 2 or better thereby not precluding them from chemotherapy trials on this basis. However, 22/92 (24\%) had such severe comorbidity that it was decided by their oncologist that they were unfit for chemotherapy. A further 16/92 (17\%) were unable to consent or died. Thus only $54 / 146$, or $37 \%$ of the total, were suitable for and were offered chemotherapy. Of these, 28/54 (52\%) declined this option and eventually only $26 / 146$ (18\% of the total) were randomised into chemotherapy trials. In patients who were fit enough and received chemotherapy, median survival was 479 days (95\% CI 421 to 537 days).

\section{DISCUSSION}

The data in this study we believe capture all cases of mesothelioma occurring in a defined geographical area for the
4 year period. All cases included in the audit were confirmed to be malignant mesothelioma on histological or cytological grounds before or after death. There is the possibility however that a few patients with a Leeds post code may have been referred to a hospital outside of Leeds. We know, however, from lung cancer studies in this area that the proportion of patients with a Leeds address referred to other hospitals outside of Leeds is less than $1 \%$. The value for mesothelioma is therefore likely to be similar. Although registry data from NYCRIS for 2005 are not yet available, mesothelioma registrations for 2002-2004 are similar to patient numbers captured in our audit population (see table 1).

In this study, recording of asbestos exposure history in the clinical record reached $86 \%$ overall. Documentation of definite or probable asbestos exposure was 87 (85\%) of 126 patients where asbestos histories were obtained and recorded. Howel et al, studying patients with malignant mesothelioma in West Yorkshire, obtained histories of likely or possible exposure in $95 \%$ of their sample from case notes or interviews with relatives of the deceased patient. ${ }^{14}{ }^{15} \mathrm{It}$ is likely therefore that the initial recording of an asbestos exposure history in medical notes may often be inaccurate. It is common, for example, for patients not to recollect exposure at the time of original consultation but at a later date.

At presentation, performance status was 1.8, median survival from the time of diagnosis 267 days (8.9 months) and median age at presentation 74 years. This compares unfavourably with the 272 case series by Yates et al in the south east of England where median survival from symptom onset was 14 months and a mean age at death of 65 years. ${ }^{3}$ In this earlier series, the authors thought they had captured almost all deaths from mesothelioma cases in the south east occurring in 1987 since this was the last year before the abolition of industrial death benefit and that in practice most cases were referred to the coroner irrespective of occupational history. This claim, however, is unsubstantiated. Age and performance status at presentation were significant poor prognostic indicators in scoring systems from the European Organisation for Research and Treatment of Cancer (EORTC). ${ }^{16}$ and the Cancer and Leukaemia Group B (CALGB) studies. ${ }^{17}$ These were validated in a 142 patient series by Edwards et al from the UK. ${ }^{4}$ Mean age at the time of diagnosis in the latter series was 64 years but selection bias may have occurred by including potentially younger patients referred through a tertiary cardiothoracic unit for radical surgical intervention. Entry into the 337 patient CALGB chemotherapy based series required a performance status of $0-1$ and the worst survival (mean 1.4 months) occurred for patients with a performance status of $1 / 2$ and white blood cell count $\geqslant 15.6 / \mu \mathrm{l}$. Based on these previous studies, our patients are disadvantaged by the fact they are a decade older and with worse performance status. We believe the poorer prognosis of this series is the result of capturing all patients in a defined geographical area. In addition, our data are consistent with the expectation that the mean age at presentation of mesothelioma is increasing because of a cohort effect of widespread asbestos exposure of men born between 1945 and 1950. ${ }^{18}$ Median survival for epitheliod, sarcomatoid or biphasic histological types was 325, 175 and 161 days, respectively. As in other series, sarcomatoid histology conferred a worse prognosis. ${ }^{4}$

The presence of pleural effusions associated with malignant pleural mesothelioma is common and problematic. It is commonly associated with chest pain, ${ }^{19}$ significantly worsens symptoms of breathlessness, reducing performance status and often requires inpatient hospital treatment, thus reducing quality of life further. The management of pleural effusion is not without risk. Complications can occur, such as tumour 
invasion of the tract, ${ }^{20} 21$ pneumothorax or introduction of infection into the chest cavity or a severe systemic inflammatory response to the pleurodesing agent. ${ }^{22}$ There is often the possibility that drainage of fluid will be ineffective because of lung encasement by the tumour, or that the effusion will quickly re-accumulate because of ineffective pleurodesis by surgical or medical means. The majority of patients received either a medical or surgical pleurodesis following drainage of a symptomatic effusion. In our population, more than twice as many were referred for a video assisted thorascopic pleurodesis. These patients had partial pleurectomies or talc poudrage.

Survival between the bedside and surgical pleurodesis groups was not significantly different but there were more recurrences in the talc slurry group. This may be because of rejection by the surgeons of those who on CT appearance were likely to have a trapped lung and those who were unfit. It is interesting that in the remainder of the patients following this selection a bedside pleurodesis conferred no statistical benefit than a chest drain only. A recent randomised trial of 501 patients showed no overall significant difference in 30 day outcomes between talc poudrage and talc slurry; however, subgroup analysis showed that patients with lung primaries had higher success rates through surgical pleurodesis. ${ }^{23}$ Further randomised trials are needed.

Patients are usually offered prophylactic radiotherapy locally to the chest wall following any interventional procedure to prevent the risk of seeding along the tract, ${ }^{7}$ the risk of such events being clinically important is unknown. The recommendation for treatment is largely based on a study by Boutin et al who, in a randomised controlled trial, compared 20 patients who received $21 \mathrm{~Gy}$ in three fractions within 10-15 days of their procedure with 20 controls. There were none versus eight metastases in the treatment versus control group, respectively. It was concluded that the incidence fell from $40 \%$ to zero as a result of treatment. ${ }^{24}$

Our experience of metastasis occurring along tracts has been different. Sixty per cent of our patients received prophylactic radiotherapy following VATS, cutting needle CT biopsies or chest drains. This rate, although poor, is above the combined rate from a series of patients collected from Northern England of $40 \%$ between 1998 and $2001{ }^{12}$ The absolute risk of a symptomatic recurrence in an unselected population is not known. In the 49 untreated patients, we had only one case of metastasis in the relevant area on the chest wall. The remaining six cases occurred in patients who had received prophylactic radiotherapy. Median time from procedure to treatment in these patients was 53 days. Only one of the six occurrences in the treated group received their radiotherapy within the 4 week maximum recommended by the $\mathrm{BTS}^{8} ; 50 \%(3 / 6)$ of those with chest wall metastasis received radiotherapy within 6 weeks of their procedure. While delay to treatment may be a reason for the ineffectiveness of prophylactic radiotherapy, it should be noted that there was only one occurrence in the untreated group. Recent support for the argument that radiotherapy is ineffectual at preventing tract invasion with tumour is given by the most recent randomised control trial by O'Rourke and colleagues. ${ }^{25}$

Complete data on the reasons for delays to prophylactic radiotherapy were not available; the most frequent examples from the available data include: delays to oncology referral, pressure on the radiotherapy service, morbidity surrounding the episode of intervention precluding rapid treatment and patients being unable to attend initial appointments. The median time to tract invasion was 174 days. Thirty-eight per cent (56/146) of our cohort had died at this point and this short survival time may explain in part why our incidence of tract invasion by tumour was low at $5 \%$. In eight other series, the mean was $19 \%$ with extremes of $2 \%$ and $51 \% .^{20}{ }^{26-32}$ We believe that the timing and benefit of prophylactic radiotherapy needs reappraisal.

Most trials of chemotherapy in mesothelioma stipulate a WHO/ECOG performance status of at least 2 or better. A significant proportion of patients with a performance status of 0-1 did not receive chemotherapy, and only two patients with a performance status of 2 were enrolled in clinical trials. This decision was made either at an MDT, which always included one of three oncologists with a declared interest in thoracic oncology, or following consultation with one of the oncologists after an MDT referral. Comorbidity was the main reason for patients being ineligible for chemotherapy. Of the patients who were offered chemotherapy $(54 / 146,37 \%)$, less than half accepted so only 26 of the original $146(18 \%)$ in this cohort actually had it. Many patients were unwilling to undergo treatment side effects with uncertain benefit, especially perhaps because at the time of diagnosis they were asymptomatic. The results of the MSO1 trial may provide evidence to help patients make the decision as to whether or not they wish to receive chemotherapy or palliative care.

In summary, this population based audit has shown that the median age of diffuse pleural malignant mesothelioma at presentation is, as expected, increasing, median survival is not improving (and may be worsening) and a large proportion of patients have a poor performance status and comorbidities at presentation precluding palliative chemotherapy. In our series, surgical thorascopic pleurodesis appeared to be associated with fewer recurrences of effusions than those who received a bedside pleurodesis but this may reflect the patient selection. Further randomised control trials are needed. Finally, the timing and role of prophylactic chest wall radiotherapy to biopsy sites needs reappraisal.

\section{Competing interests: None.}

\section{REFERENCES}

1. Ribak J, Selikoff JJ. Survival of asbestos insulation workers with mesothelioma. $\mathrm{Br} \mathrm{J}$ Ind Med 1992;49:732-5.

2. Hodgson JT, McElvenny DM, Darnton AJ, et al. The expected burden of mesothelioma mortality in Great Britain from 2002 to 2050. Br J Cancer 2005;92:587-93.

3. Yates DH, Corrin B, Stidolph PN, et al. Malignant mesothelioma in south east England: clinicopathological experience of 272 cases. Thorax 1997;52:507-12.

4. Edwards JG, Abrams KR, Leverment JN, et al. Prognostic factors for malignant mesothelioma in 142 patients: validation of CALGB and EORTC prognostic scoring systems. Thorax 2000;55:731-5.

5. Steele JPC, Klabastsa A. Chemotherapy options and new advances in malignant pleural mesothelioma. Ann Oncol 2005;16:345-51.

6. Vogelzang NJ, Rusthoven JJ, Symanowski J, et al. Phase III study of pemetrexed in combination with cisplatin versus cisplatin alone in patients with malignant pleural mesothelioma. J Clin Oncol 2003;21:2636-44.

7. Ellis $\mathbf{P}$, Davies AM, Evans WK, et al. The use of chemotherapy in patients with advanced malignant pleural mesothelioma: a systemic review and practice guideline. J Thorac Oncol 2006;1:591-601.

8. British Thoracic Society. Statement on malignant mesothelioma in the United Kingdom British Thoracic Society Standards of Care Committee. Thorax 2007;62:ii1-ii19.

9. Wolanski KD, Whitaker D, Shilkin KB, et al. The use of epithelial membrane antigen and silver stained nucleolar organizer regions testing in the differential diagnosis of mesothelioma from benign reactive mesothelioses. Cancer 1998;82:583-90.

10. Whitaker D, Shilkin KB. Diagnosis of pleural malignant mesothelioma in life: a practical approach. Pathology 1984;143:147-75.

11. Robinson BWS, Musk AW, Lake RA. Malignant mesothelioma. Lancet 2005;366:397-408.

12. Clayson H. Palliative care in mesothelioma: What are the needs of patients and their families. Thesis in preparation. The Hospice of St Mary Furness, University of Sheffield.

13. Antunes G, Neville E, Duffy J, et al. BTS guidelines for the management of malignant pleural effusions. Thorax 2003;58(Suppl II):ii29-38. 
14. Howel D, Arblaster L, Swinburne L, et al. Routes of asbestos exposure and the development of mesothelioma in an English region. Occup Environ Med 1997:54:403-9.

15. Howel D, Gibbs A, Arblaster L, et al. Mineral fibre analysis and routes of exposure to asbestos in the development of mesothelioma in an English region. Occup Environ Med 1999;56:51-8.

16. Curran D, Sahmoud T, Therasse P, et al. Prognostic factors in patients with pleural mesothelioma: the European Organisation for Research and Treatment of Cancer Experience. J Clin Oncol 1998;16:145-52.

17. Hernden JE, Green MR, Chahinian AP, et al. Factors predictive of survival among 337 patients with mesothelioma treated between 1984 and 1994 by the Cancer and Leukaemia Group B. Chest 1998;113:723-31.

18. Peto J, Decarli A, Vecchia C. La Levi F, Negri E. The European mesothelioma epidemic. Br J Cancer 1999;79:666-72.

19. Scott B, Mukherjee S, Lake R, et al. Malignant mesothelioma. In: Hanson, ed. Textbook of lung cancer. London: Martin Dunitz, 2000:273-93.

20. Elmes PC, Simpson MJC. The clinical aspects of mesothelioma. $0 \mathrm{~J}$ Med 1976:179:427-49

21. Van Ooijen B, Eggermont AMM, Wiggers T. Subcutaneous tumour growth complicating the positioning of the Denver shunt and intrapleural port-a-cath in mesothelioma patients. Eur J Surg Oncol 1992;18:638-40.

22. Lee G, Baumann MH, Maskell NA, et al. Pleurodesis practice for malignant pleura effusions in five English-speaking countries: survey of pulmonologists. Chest 2003:124:2229-38
23. Dresler C, Olak J, Herndon J, et al. Phase III intergroup study of talc poudrage vs talc slurry sclerosis for malignant pleural effusion. Chest 2005;127:909-15.

24. Boutin C, Rey F, Viallat JR. Prevention of malignant seeding after invasive diagnostic procedures in patients with pleural mesothelioma. A randomized trial of local radiotherapy. Chest 1995;108:754-8.

25. O'Rourke N, Curto GJ, Paul J, et al. A randomised controlled trial of intervention site radiotherapy in malignant pleural mesothelioma. J Eur Soc Ther Radiol Oncol 2007;84:18-22

26. De Lajartre AY, Tayot J, De Larjartre, et al. Aspects evoltifs des mesotheliomes pleuraux diffuse. Re Fr Mal Resp 1979:7:277-90.

27. Adams VI, Unni KK. Diffuse malignant mesothelioma of the pleura: diagnostic criteria based on an autopsy study. Am J Clin Pathol 1984;82:15-23.

28. Ratzer ER, Pool JI, Melamed MR. Pleural mesotheliomas: clinical experience with 37 patients. AJR Am J Roentgenol 1967;99:863-80.

29. Whitwell $\mathbf{F}$, Rawcliffe RM. Diffuse malignant pleural mesothelioma and asbestos exposure. Thorax 1971;26:6-22.

30. Brenner J, Sordillo PP, Magill GB, et al. Malignant mesothelioma of the pleura: review of 123 patients. Cancer 1982:49:2431-5.

31. Law MR, Hodson ME, Turner-Warwick M. Malignant mesothelioma of the pleura: clinical aspects and symptomatic treatment. Eur J Respir Dis 1984;65:162-8.

32. Ruffie $\mathbf{P}$, Feld R, Minkin S, et al. Diffuse malignant mesothelioma of the pleura in Ontario and Quebec: a retrospective study of 332 patients. J Clin Oncol 1989; 7:1157-68.

\section{Pulmonary puzzle}

\section{ANSWER}

From the question on page 401.

The incident occurred 4 days after accidental aspiration of isoparaffin which is used in fire eating. The normal procedure is to blow out the isoparaffin against a burning stick; however, in this case the patient inspired and aspirated the isoparaffin. This case describes an acute form of lipoid pneumonia or fire eater's lung. There have been reports of chronic aspiration of liquid paraffin where it has been used to treat constipation, particularly in children. ${ }^{1}$ Since liquid paraffin has a high viscosity, it depresses the cough reflex, facilitating aspiration.

The clinical presentation may vary from an incidental detection on the chest radiograph to acute pneumonia as in this case or chronic fibrosis. Inflammatory markers and white cell count are raised in the cases described. The chest radiograph typically shows alveolar consolidation and ground-glass opacities. ${ }^{2}$ A CT scan of the thorax may demonstrate areas of alveolitis, consolidation and pneumatoceles. ${ }^{3}$ Bronchoscopy and bronchoalveolar lavage may be useful where the diagnosis is in doubt. Fat globules are found in the bronchoalveolar fluid and lipidladen macrophages are noted on cytological examination. Pulmonary function testing may demonstrate bronchial hyperresponsiveness and a restrictive ventilatory defect. ${ }^{4}$

Case reports have described treatment with corticosteroids, although there are no experimental data supporting their role. ${ }^{5}$ Prophylactic antibiotics are also reported to be used in the treatment and may treat any co-existing bacterial infection. The prognosis is generally good, but one study found that $21 \%$ of patients developed complications which included fibrosis, abscesses, effusions, bronchopulmonary fistula and bacterial superinfection. ${ }^{3}$

Thorax 2008;63:439. doi:10.1136/thx.2007.090001a

\section{REFERENCES}

1. Kameswaran M, Annobil SH, Benjamin B, et al. Bronchoscopy in lipoid pneumonia. Arch Dis Child 1992;67:1376-7.

2. Gondouin A, Manzoni PH, Ranfaing E, et al. Exogenous lipoid pneumonia: a retrospective multicentre study of 44 cases in France. Eur Respir J 1996;9:1463-9.

3. Franquet T, Gómez-Santos D, Giménez A, et al. Fire eater's pneumonia: radiographic and CT findings. Comput Assist Tomogr 2000:24:448-50.

4. Brander PE, Taskinen E, Stenius-Aarniala B. Fire-eater's lung. Eur Respir J 1992;5:112-4.

5. Mullin LS, Ader AW, Daughtrey WC, et al. Toxicology update isoparaffinic hydrocarbons: a summary of physical properties, toxicity studies and human exposure data. J Appl Toxicol 1990:10:135-42. 